\title{
REPRODUCCION INDUCIDA DE “Boquichico" Prochilodus nigricans CON Gn-RH (a) EN SAN MARTIN-PERU
}

\section{GILBERTO ASCON DIONICIO*}

\section{RESUMEN}

El presente trabajo, realizado en la Estación de Pesquería "A huashiyacu", Provincia y Departamento de San M artín en el período de enero a diciembre de 1990, muestra la eficacia del "Conceptal", análogo sintético de la hormona Gn-RH; en el desove de "Boquichico" Prochilodus nigricans, A gassiz, 1829, utilizando para la dosificación hormonal dos tratamiento, el primero con $2,6 \mathrm{ml}$ de conceptal/kg. pv y el segundo con $1.3 \mathrm{ml}$ de conceptal $/ \mathrm{kg}$. pv con 3 y 2 réplicas respectivamente. Se logró el desove con el primer tratamiento alcanzándose una supervivencia de al evinos de $40 \%$.

Palabras claves: Reproducción inducida, Prochilodus nigricans, Análogo S intético, Gn-RH

\section{INTRODUCCION}

El boquichico Prochilodus nigricans (A gassiz, 1829), por su calidad de carne, rápido crecimiento y gran aceptación en el publico consumidor, es una de las especies nativas de la A mazonía Peruana, tan importante como otras para el desarrollo de la Piscicultura tropical, como Colossoma macropomum, Piaractus brachypomum, Brycon sp., etc.

El "boquichico" P. nigricans, es una especie de la familia Prochilodontidae, reofílico y su reproducción lo realiza cada año al inicio de la creciente de los ríos (Alcántara, 1980); es iliófago, de desove total, alcanzando su madurez sexual al año, (Campos, 1988).

La mayoría de investigaciones sobre reproducción inducida, se han realizado con pituitaria de peces, principalmente de "carpa" Ciprinus Carpio y boquichico Prochilodus nigricans. Sin embargo otros investigadores han realizado trabajos utilizando hormonas o análogos sintéticos a la LG-RH, como Harvey y

\footnotetext{
Instituto de Investigaciones de la A mazonía Peruana (IIA P)-Centro Regional de Investigaciones San M artín. apartado 179. Tarapoto - Perú.
} 
Hoar (1980), Wornorovich, E. (1981) y Donalson (1975) citados por Breton et al (1983).

El objetivo del presente trabajo fue determinar el efecto de la hormona de uso veterinario "conceptal" análogo sintético de la hormona Gn- $\mathrm{RH}$, en el desove de boquichico Prochilodus nigricans, con una dosis de $2.6 \mathrm{ml} / \mathrm{kg} \mathrm{p.v}$ equivalente a $0.014 \mathrm{mg} / \mathrm{kg} \mathrm{p}$.v de huserelina considerada como la dosis óptima o patrón en el desove de paco y gamitana. Se usó una dosis de $1.3 \mathrm{ml} / \mathrm{kg}$ p.v. como tratamiento de comparación.

\section{MATERIALES Y METODOS}

El presente trabajo se efectuó en la Estación de Pesquería de "A huashiyacu", Provincia y Departamento de San Martín-Perú, en el período de enero a diciembre de 1990. Los reproductores utilizados fueron traídos de la ciudad de Pucallpa al estado de alevinos en 1988 y criados en estanques seminaturales de $500 \mathrm{~m} 2$ junto con gamitanas reproductoras, proporcionándoles alimento con un nivel de proteínas del $25 \%$, recibiendo el estanque una fertilización con gallinaza a razón de $300 \mathrm{~kg} / \mathrm{ha} /$ día 3 veces por semana.

Se realizaron 5 experimentos utilizándose 2 machos por cada hembra, excepto en el primero que fue un macho por una hembra. La selección de los reproductores se hizo de acuerdo a las recomendaciones de W oynarovich (1986) y Fontenele (1959). Para el tratamiento hormonal se colocaron en estanques de concreto $(2 \times 1 \times 0.74 \mathrm{~m})$ revertidos con mayólicas, por separado machos de hembras.

Se utilizaron los siguientes materiales; calcales de tela de algodón, toallas, una mesa portátil, cochoneta forrada con plástico, jeringas descartables de 5 cc. y "conceptal' producto de uso veterinario análogo sintético de la hormona $\mathrm{Gn}-\mathrm{RH}$

A dquirido en el mercado local en frascos de $10 \mathrm{ml}$ cada uno. 
TABLA 1. Peso de los reproductores y dosificación para el desove de "boquichico' Prochilodus nigricans, mediante el uso de "Conceptal"

\begin{tabular}{|c|c|c|c|c|}
\hline SEXO & $\begin{array}{l}\text { PESO } \\
(\mathrm{kg})\end{array}$ & $\begin{array}{l}\text { DOSIFIC } \\
\text { 1 ra. Dosis } \\
(\mathrm{ml} / \mathrm{pez}) \\
(10 \%)\end{array}$ & $\begin{array}{l}\mathrm{UN}_{2 \mathrm{da}} \text { Dosis } \\
(\mathrm{ml} / \mathrm{pez}) \\
90 \%\end{array}$ & $\begin{array}{l}\text { TOTAL } \\
\text { (ml/pez) } \\
100 \%\end{array}$ \\
\hline $\begin{array}{l}\mathrm{h} \\
\mathrm{m} \\
\mathrm{h} \\
\mathrm{m} \\
\mathrm{h} \\
\mathrm{m} \\
\mathrm{h} \\
\mathrm{m} \\
\mathrm{h} \\
\mathrm{m}\end{array}$ & $\begin{array}{l}1.20 \\
0.80 \\
1.15 \\
0.75 \\
1.10 \\
0.75 \\
1.00 \\
0.38 \\
1.30 \\
0.83\end{array}$ & $\begin{array}{l}0.312 \mathrm{~T}-1 \\
0.3 \mathrm{~T}-1 \\
0.286 \mathrm{~T}-1 \\
0.130 \mathrm{~T}-2 \\
0.690 \mathrm{~T}-2\end{array}$ & $\begin{array}{l}2.808 \\
0.200 \\
2.700 \\
0.195 \\
2.574 \\
0.195 \\
1.000 \\
0.0494 \\
1.000 \\
0.107\end{array}$ & $\begin{array}{l}3.120 \\
0.200 \\
3.000 \\
0.195 \\
2.860 \\
0.195 \\
1.3 \\
0.0494 \\
1.69 \\
0.107\end{array}$ \\
\hline
\end{tabular}

El desove e incubación se realizó de acuerdo a las recomendaciones de Woynarovich (1986) y para la crianza de larvas se siguio las recomendaciones de

A scón (1990). Se instalaron japas de tela horganza de $(1.5 \times 1 \times 0.5 \mathrm{~m})$ dentro de estanques de concreto de $15 \mathrm{~m} 2$ con una renovación constante de agua. L as larvas se sembraron y permanecieron en las japas por un período de 4 días para luego pasar a los estanques de tierra previamente preparados y con abundante alimento vivo, por un período de 40 días, en que se realizó la evaluación de los alevinos. 


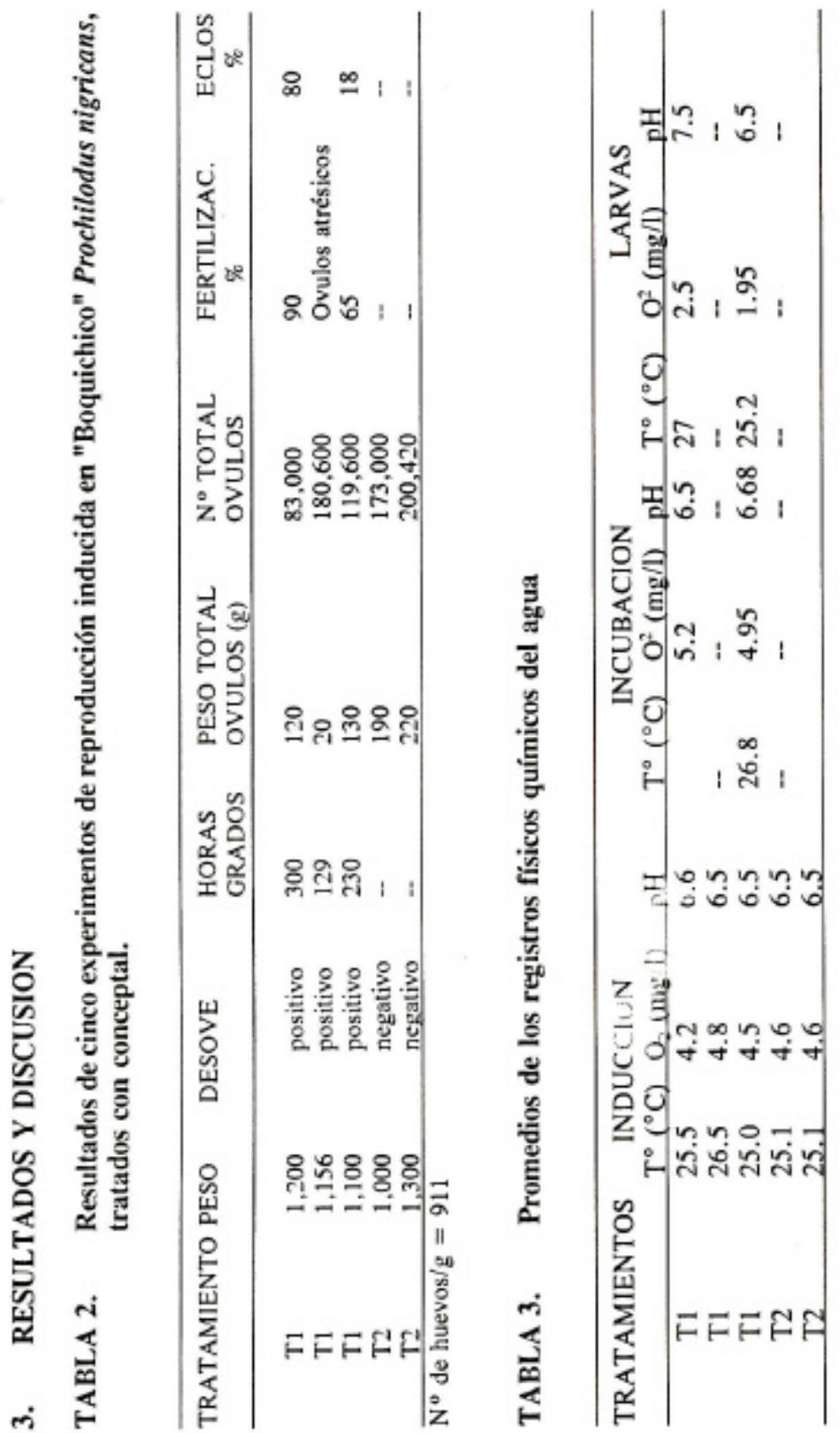


Muchos autores manifiestan, que actualmente existen análogos estructurales sintéticos de la LH-RH, algunos de los cuales poseen una vida mas larga y son mas potentes en los mamíferos que la LH-RH. Tal es así que, autores como Preto, (1983) utilizando el análogo sintético DES-GLY-IOD-SLAG-LH$\mathrm{RH}$, hormona liberadora de la LG-RH, obtiene el desove de C'olossoma macropomum aplicando una dosis de $2 \mathrm{mg} / \mathrm{kg}$ p.v.; Breton, (1983) administrando LH-RH (a) en carpa común aumentan el nivel de la hormona gonodotrópica en plasma. Ascón (1990) obtiene el desove de "paco" P. brachypomus y "gamnitana" C. macropomum cuando utiliza el "conceptal" análogo sintético de la hormona $\mathrm{Gn}$-RH de uso veterinario aplicando $2.6 \mathrm{ml} / \mathrm{kg} \mathrm{p.v}$. equivalente a (0.014 mg. de Buserelina/kg p.v.) en2 dosis, del $10 \%$ y $90 \%$ dcl total con un intervalo de tiempo de 12-14 hrs.

Los resultados del presente trabajo nos muestran el desove de "boquichico" cuando se aplicó $2.6 \mathrm{ml} / \mathrm{kg}$ p.v. y no cuando se utilizó $1.3 \mathrm{ml} / \mathrm{kg}$ p.v. Estos resultados confirman la eficacia del "conceptal" en el desove de P. nigi-icans cuando se aplica la dosis óptima o patrón (2.6 ml/kg p.v.), establecida por A scón (1990), en el desove de "paco" y "gamitana".

Las horas grado para el desove fue de 219.6 en promedio, resultados muy similares a las de Samanez (1984) que fue de 240.5 horas.

Los machos respondieron bien; asegurando una perfecta coordinación con la liberación de los óvulos. Esto concuerda con lo reportado por Harvey y Hoar (1980) observándose que la inducción a los machos es para el adelgazamiento seminal así como asegurar una coordinación en la liberación de los gametos.

El número de óvulos/g fue 911 en promedio, similar a los obtenidos por Eckman (1980).

El período de incubación de los huevos osciló de 18-21 horas a una temperatura de $25-26.80 \mathrm{C}$, estos resultados son similares a los obtenidos por Ruíz (1987) donde el período promedio de incubación duró 1745 horas, a una temperatura de $25.4 \stackrel{\circ}{ } \mathrm{C}$ y de Pezo y Sicchar (1974) que fue de 8.0 horas. a 26.5 oC.

Los resultados nos muestran también que el porcentaje de eclosión fue bajo con respecto al porcentaje de fertilización. Esto se debió a que se utilizaron para este proceso incubadoras verticales, que por no ser funcionales presentaban una pérdida considerable de huevos así como de larvas y no las de tipo Woynarovich (1981) que son las más recomendables.

A esto se sumaron otros factores como turbidez a causa de las constantes precipitaciones, impidiendo de esta forman obtener mayor número de larvas. La evaluación realizada a los alevinos a 40 días de edad en uno de los desoves representa una supervivencia del $40 \%$, a diferencia de investigaciones realizadas por otros autores como Pezo y Sicchar (1974), Eckman (1980), Ruíz (1987) y Samanez 
(1984) donde se llegó hasta la fase de larvas; muriendo generalmente a los 156 10 días de haber sido eclosionadas.

Los registros físico-químicos se muestran en el Cuadro 3 donde se puede ver poca variación en las tres fases (Inducción, Incubación y larvas).

Por otro lado el autor concuerda con lo manifestado por Bermódez (1979) y Kossowsky (1980) quienes afirman que es mejor utilizar hormonas de tipo sintético, ya que el uso de glándulas de especies exóticas que se ofrecen en el mercado es iscutible por desconocer entre otros, su procedencia, el tiempo de almacenamiento, etc.

\section{BIBLIOGRAFIA}

ALCANTARA, F. y H. GUERRA, 1990. Aspectos de alevinaje de las principales especies nativas utilizadas en piscicultura en la Amazonía Peruana. Folia A rnaz. 2:135-140.

ASCON, G., 1988. Producción de alevinos de peces de consumo por medio de la reproducción artificial en Selva Alta Rey. Lat. A cuil. NO 36. LimaPerú.

-----1989. Reproducción artificial inducida de gamitana, paco y boquichico con el uso de conceptal análogo sintético de la hormona (Gn-RH). Informe Técnico Interno IIAP-San M artín IOp.

----1990. Cultivo masivo de rotíferos y cladóceros para la crianza de larvas de peces del género Colossoma en estanques de cemento. Folia A mazon. 2: 135- 140.

BERMUDEZ, D. 1979. Observaciones sobre el desarrollo embrionario de "cachana" Colossoma macropomum cuvier 1818. Estación de Piscicultura, U niversidad Centro Occidental, Barquisimeto, V enezuela.

BRETON, B. et al. 1983. Efecto of synthetic LH-RH Analog on Plasma Gonodotropin Levels and maturational response to 17 - Hydroxy-108 dihydroprogesterone. Elsevier scienti tic puhlishing aquacultures, 32 (1983) 105-114-A msterdam.

CAM POS, L., 1988. M anual de Piscicultura Tropical-IIA P-Iquitos-perú. 114 p. 
CAMPOVERDE, L; G. SALDAÑA; G. ASCON; y R, LOAYZA, 1987. Efectividad de la Gonadotropina Coriónica humana en la reproducción de "gamitana" Colossoma macropomum (CUVIER) 1818 en el Departamento de San M artín. Hidrobios XI (1-2): 1-7, 1987 U niversidad de Trujillo-Perú.

CAMPO VERDE, L. et al. 1987. Efectividad de la gonadotropina coriónica humana en la reproducción de "gamitana" Colossoma macropomum (Cubier) 1818 en el Departamento de San M artín. Hidrobios XI (1-2) : 1-7, Universidad Nacional de Trujillo-Perú.

FONTENELE, 0. 1959. Inyecting pituitary (Hypophyseal) hormones into tish to induced spawning Dept. Nac. Obras contra as secas, servicio de Piscicultura № 68-Brasil.

GERALDO, B. y A. FERRARI, 1986. Inducción para ovulación de Tambaqui Colossoma macropomum, usándo dosis análogos de LH-RH, sintese dos trahalhos realizados con especies do Genero Colossoma-CEPTAMarzo/82 A A bril/86. S.P., Brasil.

HARVEY, J. y 5. HOAR, 1980. Teoría y práctica de la reproducción inducida en los peces. Concejo $\mathrm{N}$ acional de Investigaciones $\mathrm{C}$ anadá, $480 \mathrm{p}$.

KOSSOWSKI, C., 1980. Esanyo de reproducción inducida en "Palometa Carachica" en Mylossoma duriventris (cuvier) 1818 (pisces cypriniformes) con el uso de G.C.H. Estación de Piscicultura, Escuela de Agronomía Universidad Centro Occidental, Barquisimento V enezuela, A cta Cient. Venezolana, 31:444-448.

PEZO, R. y L. SICCHAR, 1979. Reproducción inducida por hipofisación en "boquichico" Prochilodus nigricans A gassiz, 1829. Tesis para obtener el Título de Biólogo Universidad Nacional de la Amazonía Peruana. Iquitos, Perú.

PRETO, R., 1983. Reproducción de Colossoma macropomuin, con la hormona LH RH análogo Luteinizing homona realeasing homona Desgly-IODLARG-LH RH-Ethylamide A nálogue. Estación Dulceacuícola de Divisa. Panamá. Rey. - Lat. A cui. No 18-Lima-Perú. 
RUIZ, L.; A. SAENZ; 1. LEON; y C. VILLANUEVA; 1987. Reproducción de "boquichico" Prochilodus nigricans Agassiz 1829) por medio de hormonas, Rey. Lat. A cui. No 31 Lima-Perú.

RUIZ, L. et al. 1987. Reproducción de "boquichico" Prochilodus nigricans (A gassiz 829) por medio de hormonas, Rey. Lat. Acui. N0 31 LimaPerú.

SAMANEZ, 1., 1985. Reproducción inducida de "gamitana" C. macropomum, "boquichico $P$. nigricans y sábalo cola roja $B$. erytropterum. Informe Técnico anual. Conv. IIA P-IV ITA. Pucallpa-Perú.

SALDAÑA, G. y G. ASCON, 1986. Ensayo sobre reproducción inducida de "gamitana" C'. macropomum (Cuvier) 1818, con el uso de Gonadotropina Coriónica Humana $(\mathrm{GCH})$ Rey. Hidrobios X: 1-12 U niversidad Nacional de Trujillo. Trujillo-Perú.

WOYNAROVICH, E. y L. HORVATH, 1981. Propagación artificial de peces de aguas templadas, Manual para Extensionistas. FA O. Doc. Técn. PESCA (201).

WOY NAROVICH, E., 1986. Tambaqui e Pirapitinga propagación artificial e criacas de alevinos compahía da D esenvolvimiento do vale de Sao Francisco (CODEVASF) Brasil-68p. 